\title{
Peertechz
}

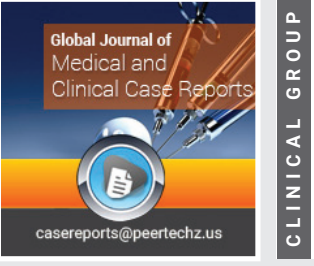 \\ Medical and Clinical Case Reports argumecess}

\section{Atypical presentation of a fish bone foreign body: A case report and review of the literature}

\author{
Rabab R Bagdadi' and Leena R Baghdadi ${ }^{2 *}$ \\ ${ }^{1}$ Consultant Radiologist, Department of Diagnostic Radiology, East Jeddah Hospital, Ministry of \\ Health, Jeddah, Saudi Arabia \\ ${ }^{2}$ Assistant Professor and Clinical Epidemiologist, Department of Family and Community Medicine, \\ College of Medicine, King Saud University, Riyadh, Saudi Arabia
}

Received: 14 April, 2021

Accepted: 22 April, 2021

Published: 23 April, 2021

*Corresponding author: Dr. Leena R Baghdadi, Assistant Professor and Clinical Epidemiologist, Department of Family and Community Medicine, College of Medicine, King Saud University, Riyadh, Saudi Arabia, Tel: +966 12 2327555; Fax: +966 11 2125050; E-mail: lbaghdadi@ksu.edu.sa,drleena.baghdadi@gmail.com ORCID: http://orcid.org/0000-0003-3315-6266

Keywords: Fish bone; Foreign body; Lower airway; Computerized tomography; Case report

https://www.peertechzpublications.com

Check for updates

\begin{abstract}
Introduction: Fish bone Foreign Body (FFB) accidental ingestion is a most common presenting complaint for patients attending the emergency department. It is more frequently lodged within the oropharynx and the oesophagus. However, fish bone impacting in the respiratory system is extremely rare; accounting for $14 \%$ of reported cases, with approximately few cases reported in the literature involved the lower airway.

Patient concern: A 27- year-old man presented clinically as a case of chest infection with a 5-day history of palpitation, right pleuritic chest pain and shortness of breath.

Diagnosis: We report a case of atypical presentation of FFB ingestion. Although patients with foreign bodies' ingestion sometimes presented with cough, there are a wide spectrum of clinical symptoms, especially those with lower airway FFB; where imaging findings are not usually spotted in the emergency department to point to the diagnosis.

Intervention: Swab for COVID-19 was negative and the initial chest X-ray showed poor inspiratory effort and no air space opacity. Although CT pulmonary angiography for pulmonary embolism was negative, an incidental finding suggestive of foreign body was reported in the right lower lung region.

Outcomes: The foreign body was distal within the lung parenchyma; therefore, there was no need for urgent surgical intervention and there should be a 1-month outpatient clinic follow-up and patient was discharged on antibiotic.

Conclusion: Although fish bone ingestion is a common food related foreign body ingestion, cases can often present a diagnostic dilemma and can be easily misdiagnosed or mistaken for other diagnoses. Therefore, vigilant and thorough history taking, and the consideration of a FFB in the differential diagnosis is essential.
\end{abstract}

\section{Abbreviations}

FFB: Fish Bone Foreign Body; ER: Emergency Room; CT: Computerized Tomography; ECG: Electrocardiogram

\section{Introduction}

Foreign body ingestion is a common presentation in the Emergency Room (ER). Fish Bone Foreign Body (FFB) ingestion is the most common cause of accidental food-associated foreign body ingestion (84\%) [1] in the Mediterranean and Asian populations, especially in the coastal areas [2]. The majority of these bones pass through the gastrointestinal tract without symptoms or complications, and $<5 \%$ of the patients with accidentally ingested fish bones develop complications $[2,3]$.

Here, we report one of the first cases of an atypical location of an ingested fish bone, impacting the lung parenchyma, which was visualized by a computerized tomography (CT) scan. This case report highlights the importance of vigilant and thorough history taking and a physical examination not only by emergency physicians, but also specialists including, gastroenterologists, cardiothoracic surgeons and radiologists.

\section{Case report}

A 27-year-old man with an unremarkable medical 
history presented to the Respiratory Zone in the Emergency Department with a 5-day history of palpitation, right pleuritic chest pain, shortness of breath, and no documented fever. The swab for COVID-19 was negative, 1 day before the presentation. As his swab was negative and his clinical presentation was not suggestive for COVID-19, he was transferred to the Emergency Department for further investigations. On examination, he had stable vitals, although his heart rate was 136 beats/min. A chest examination revealed decreased air entry on the right side and the electrocardiogram (ECG) showed sinus tachycardia. An initial chest $\mathrm{X}$-ray was performed in the supine position (Figure 1) and the main concern at that time was to exclude the presence of a COVID-19-related chest infection as his symptoms were suggestive of chest problems. Therefore, the ingested foreign body diagnosis was initially missed. The patient was seen by the Emergency Department specialist and discharged from the Respiratory Zone to the Emergency Department as the COVID-19 swab was negative.

In the Emergency Department, laboratory investigations revealed a high D-dimer of $1540 \mathrm{\mu g} / \mathrm{ml}$ and normal white blood cells $11.17 \times 10^{3} / \mu \mathrm{L}$, hemoglobin $12.6 \mathrm{~g} / \mathrm{dL}$, platelets $296 \times 10^{3} / \mathrm{uL}$, international normalized ratio 1.18 , and creatinine $1.2 \mathrm{mg} / \mathrm{dl}$. The ECG showed Q3 T3 changes and he was tachypneic. The CT pulmonary angiography for pulmonary embolism was negative (Figure 2). However, there was an incidental finding in the right lower lung; there was a $2.8-\mathrm{cm}$, long hyperdense linear structure of osseous density with adjacent tiny foci of similar density and a surrounding consolidation suggestive of a foreign body, likely to be a fish bone (Figures 3a,b-5a,b). The patient was referred for an emergency bronchoscopy and an evaluation by the thoracic surgeon. The opinion was that the foreign body was not the cause of the patient's symptoms as it was distal within the lung parenchyma; therefore, there was no need for urgent surgical intervention and there should be a 1-month outpatient clinic follow-up. He was discharged with antibiotics (for 10 days duration) for a chest infection and an appointment for the follow-up in the outpatient clinic. The management plan was discussed with him and his perspectives were taken in consideration. He was followed up at 1 month

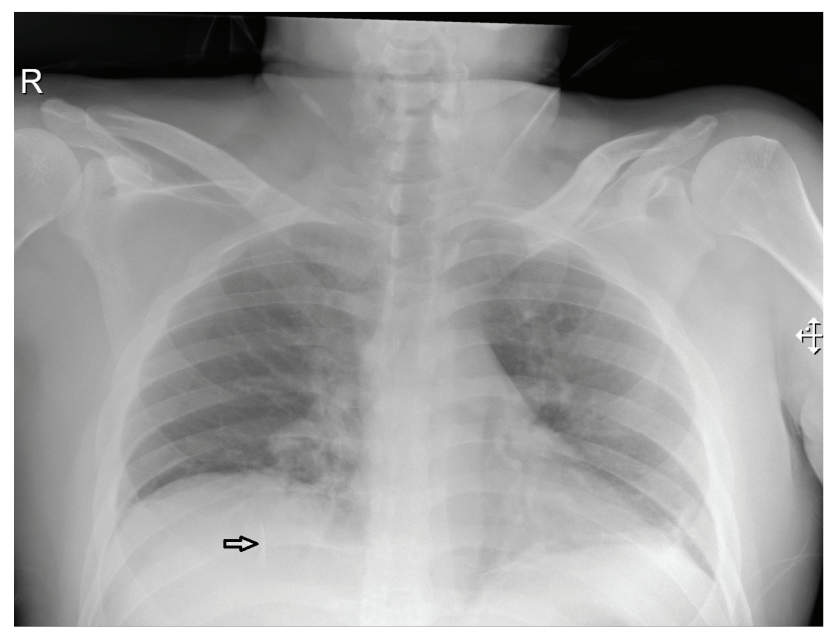

Figure 1: Initial chest X-ray showed poor inspiratory effort and no air space opacity. A linear hyperdensity is noted in the right lower lung, which was initially missed.

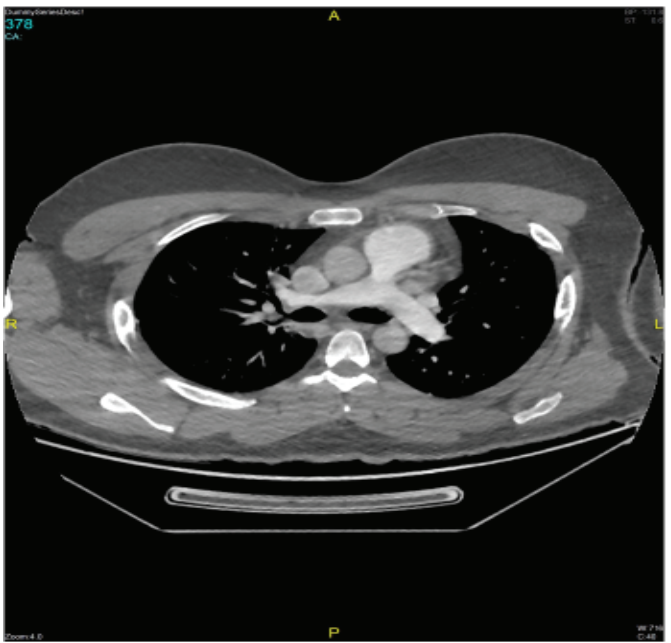

Figure 2: An axial cut of the computerized tomography pulmonary angiogram demonstrating complete opacifications of the pulmonary trunk and the major pulmonary arteries with contrast, no filling defects.
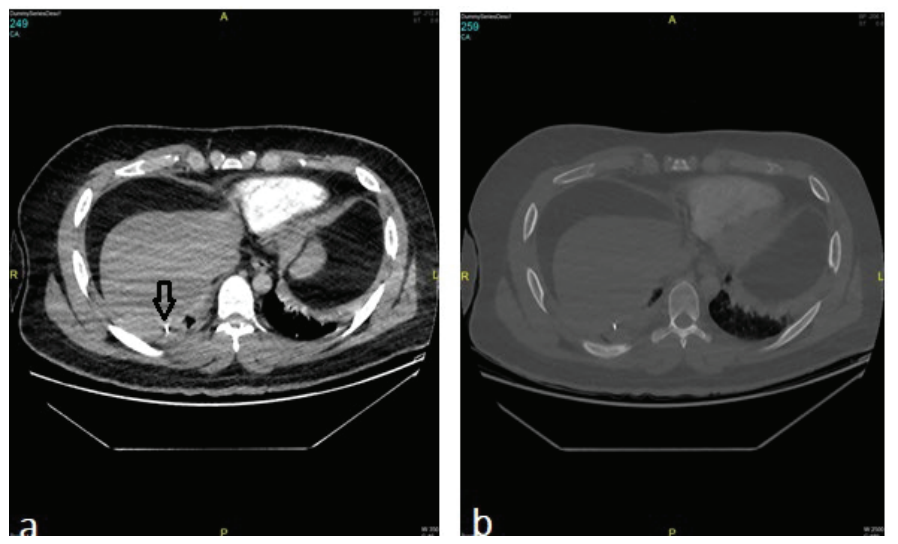

Figure 3: Axial computerized tomography scan of the chest.

a. Soft-tissue window

b. The bone window

Both views revealed the incidentally detected hyperdense structure within the right lower lung parenchyma with streak artifacts (Note the similar density to the adjacent osseous structures in b).

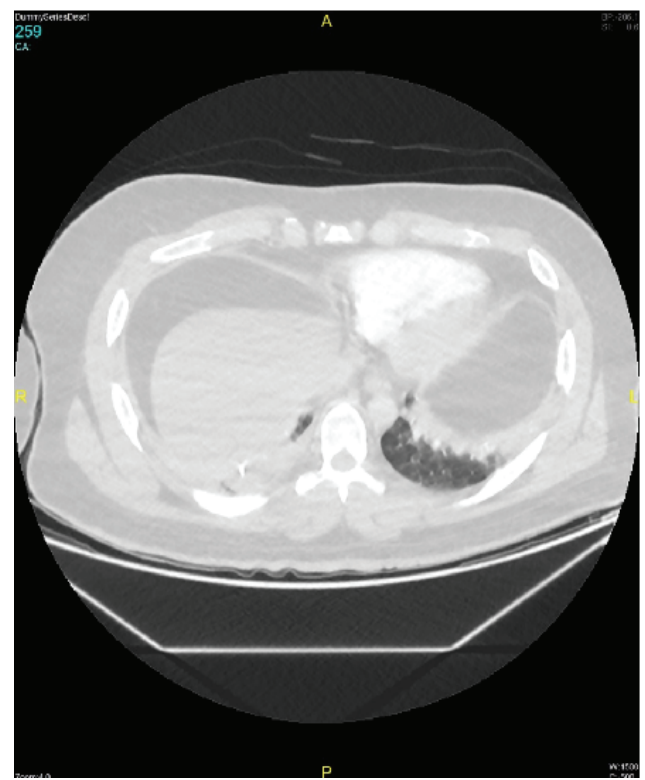

Figure 4: Axial computerized tomography scan of the chest. The lung window demonstrates the surrounding area of consolidation in the right lower lung.

Citation: Bagdadi RR, Baghdadi LR (2021) Atypical presentation of a fish bone foreign body: A case report and review of the literature. Glob J Medical Clin Case Rep 8(1): 034-037. DOI: https://dx.doi.org/10.17352/2455-5282.000123 
and 2 months, he was doing well and his initial complaints that he presented at the emergency department were resolved with no new complaints. The chest $\mathrm{X}$-ray was repeated and revealed an unchanged appearance of the linear hyperdense foreign body in the right lower lung (Figure 6). All the ethical protocols were followed, and the patient gave informed consent for publication of this case report. The patient's information was de-identified.

\section{Discussion}

Accidental FFB ingestion is a common ER presentation in geographical areas where regular consumption of unfilleted fish is popular. Although the age distribution of FFB ingestion varies, the highest prevalence is in young children (aged 1-11 years) and middle-aged adults (aged 30-59 years old) [4,5]. In adults, the majority of FFB are impacted in the oropharynx and the esophagus (about 86\%) [6], and are largely expelled from the gastrointestinal tract without intervention. However, 10$20 \%$ of cases need emergency endoscopic removal [1]. Although an FFB rarely migrates to the lower respiratory system [1], tracheobronchial foreign body aspiration has been reported in $14 \%$ of FFB cases [4]. Typically, the lower respiratory system
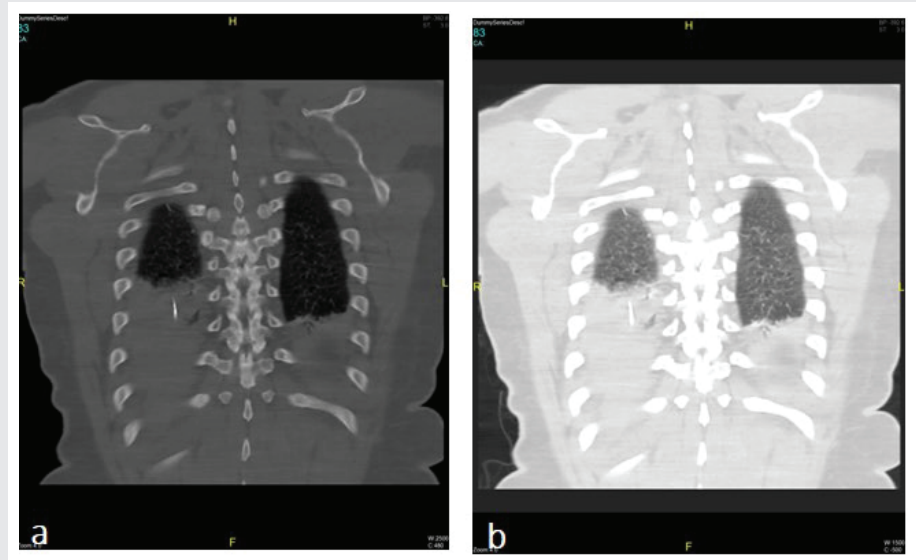

Figure 5: Coronal views of the chest on a computerized tomography scan.

a. The bone window shows the linear hyperdense foreign body in the right lower lung with surrounding consolidation.

b. The lung window shows the right lower lobe consolidation surrounding the hyperdense foreign body.

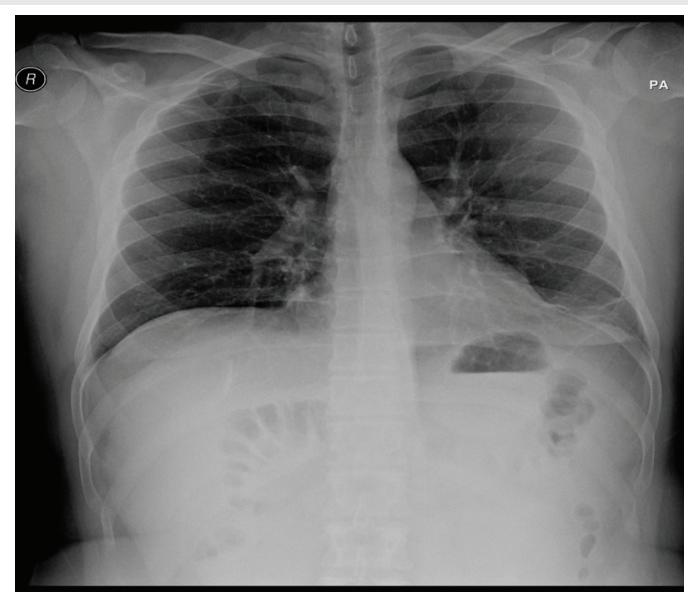

Figure 6: The follow up chest X-ray revealed an unchanged appearance of the linear hyperdense foreign body in the right lower lung. involvement occurs in adults with underlying neurological or medical conditions [7]. In the present case report, however, we reported an atypical presentation of FFB aspiration in a healthy man aged 27 years. Due to the nonspecific clinical presentation, adult lower airway FFB aspiration is easily misdiagnosed.

Evidence about direct presentation of lower airway FFB impaction is limited. The majority of the reported literature showed the involvement of the lungs secondary to a longstanding FFB impaction and complete esophageal wall penetration, which led to a lung abscess, recurrent pneumonia, tracheoesophageal fistula, empyema, and pneumothorax [4]. Additionally, FFB impaction has caused lung collapse and obstructive pneumonia [1]. These severe complications are associated with poor prognosis; and depend on the presence of many risk factors. Lower airway FFB is uncommon in adults and usually obscure or indirect. In fact, eating habits can influence FFB impaction in the lower airway. Habits vary between countries and have been found to be different in Chinese adults compared to Western adults [8]. Worldwide, fish fin has been thought to be inedible. However, it is popular in China where Chines like the taste of the flesh surrounding the fin $[4,8]$. Other risk factors include the bone type and length of the bone $(>3 \mathrm{~cm})$ [4]. A long time lapse after FFB ingestion can predispose the patient to lower airway FFB impaction. An 80-year-old Chinese woman had an aspirated fish fin that migrated into the lower airway and remained undiagnosed for 3 years [9]. If left untreated, this FFB in Chinese woman could have caused several lung complications. A lung abscess, although rarely induced by an FFB, was reported in a middleaged woman who had a continuous dry cough and fever [10].

Our patient presented with atypical symptoms of an FFB ingestion during the COVID-19 pandemic. His respiratory symptoms were typical of a pulmonary embolism. Based on the patient's presenting symptoms, a primary imaging modality is a simple chest X-ray, which was followed by a CT pulmonary angiography to rule out the presence of a pulmonary embolism. Although simple radiography has low sensitivity, if severe complications or perforations are present, ordering a CT scan for every suspected FFB is not recommended [11]. CT scans are expensive and have high false-negative rates; they expose the patients to unnecessary high radiation [12]. Imaging patients with ambiguous or atypical symptoms, including geriatric $[8,13,14]$, and young healthy patients [13] has little diagnostic value [15]. However, the benefits and risks of a CT scan are highly individualized. Some severe cases with a clear history of FFB ingestion do mandate a CT scan as FFB ingestion is usually difficult to identify on a plain chest X-ray [16]. For example, an FFB ingestion was revealed when a non-contrast CT scan was performed to diagnose a patient with severe retrosternal chest pain with a clear history of accidental FFB ingestion 5 days before the symptoms. The scan revealed a bilateral cystic bronchiectasis with a 20-mm-long and 2-mm-thick hyperdense fish bone. Almost $95 \%$ of the FFB penetrate the posterior wall of the esophagus. Therefore, a chest CT scan with contrast revealed the complication of the FFB, which was a bronchial artery pseudoaneurysm developing from a hypertrophied right bronchial artery that was in close contact with the fish bone [2]. 


\section{Conclusion}

We reported an unusual case of FFB aspiration into the lower airway. Familiarity with an atypical presentation of clinical history and various imaging features can establish the diagnosis of accidental FFB ingestion. The lack of background history about ingesting the fish bone and unfamiliarity with the appearance of the FFB in the chest X-ray complicate its diagnosis. An initial diagnosis of FFB in the lower airway can be difficult due to the nonspecific signs and symptoms and CT scans have high false-negative rates. Vigilant and thorough history and a physical examination should be taken by physicians at the Emergency Department to prevent unnecessary complications of FFB.

\section{Acknowledgment}

Special thanks for support by the College of Medicine Research Center, Deanship of Scientific Research, King Saud University, Riyadh, Saudi Arabia. Special thanks for Dr Fatima Alshehri, a Radiology Senior Registrar at East Jeddah Hospital, Ministry of Health, Jeddah, Saudi Arabia, for accepting the case for the CT scan, reading, and writing the report.

\section{References}

1. Venkatesh SH, Venkatanarasimha Karaddi NK (2016) CT findings of accidental fish bone ingestion and its complications. Diagn Interv Radiol 22: 156-160. Link: https://bit.ly/2QqjodR

2. Matrella F, Lhuaire M, Piardi T, Dokmak S, Bruno O, et al. (2014) Liver hilar abscesses secondary to gastrointestinal perforation by ingested fish bones: surgical management of two cases. Hepatobiliary Surg Nutr 3: 156-162. Link: https://bit.ly/2QfVYba

3. Mathew RP, Jayaram V, Toms A, Joshi M (2020) Fish bone induced bronchial artery pseudoaneurysm in a patient with underlying bronchiectasis-a case report. Egyptian Journal of Radiology and Nuclear Medicine 51: 128. Link: https://bit.ly/3sBIMKx

4. Kim HU (2016) Oroesophageal Fish Bone Foreign Body. Clin Endosc 49: 318326. Link: https://bit.ly/3elolqJ
5. Kim JP, Kwon OJ, Shim HS, Kim RB, Kim JH, et al. (2015) Analysis of Clinical Feature and Management of Fish Bone Ingestion of Upper Gastrointestinal Tract. Clin Exp Otorhinolaryngol 8: 261-267. Link: https://bit.ly/3axmeo5

6. Arulanandam S, Das De S, Kanagalingam J (2015) A prospective study of epidemiological risk factors for ingestion of fish bones in Singapore. Singapore Med J 56: 329-332 quiz 33. Link: https://bit.ly/3aw9UVc

7. Boyd M, Chatterjee A, Chiles C, Chin R (2009) Tracheobronchial foreign body aspiration in adults. South Med J 102: 171-174. Link: https://bit.ly/3tJ5a62

8. Chen CH, Lai CL, Tsai TT, Lee YC, Perng RP (1997) Foreign body aspiration into the lower airway in Chinese adults. Chest 112: 129-133. Link: https://bit.ly/3sL72Kd

9. Lin CY, Huang SF, Lan CC, Wu YK, Huang CY, et al. (2013) Fish fin aspiration: an unusual type of lower airway foreign body in a Chinese adult. Respir Care 58: e20-e22. Link: https://bit.ly/3arNeW4

10. Tan S, Tan S, Peng M, Yu F (2015) Management of an ingested fish bone in the lung using video-assist thoracic surgery: a case report. Medicine (Baltimore) 94: e943. Link: https://bit.ly/3ethOjw

11. Eisen GM, Baron TH, Dominitz JA, Faigel DO, Goldstein JL, et al. (2002) Guideline for the management of ingested foreign bodies. Gastrointest Endosc 55: 802-806. Link: https://bit.ly/3nhwLJh

12. Fletcher JG, Kofler JM, Coburn JA, Bruining DH, McCollough CH (2013) Perspective on radiation risk in CT imaging. Abdom Imaging 38: 22-31. Link: https://bit.ly/3sGy59|

13. Lin L, Lv L, Wang Y, Zha X, Tang F, et al. (2014) The clinical features of foreign body aspiration into the lower airway in geriatric patients. Clin Interv Aging 9: 1613-1618. Link: https://bit.ly/3elpgNj

14. Baharloo F, Veyckemans F, Francis C, Biettlot MP, Rodenstein DO (1999) Tracheobronchial foreign bodies: presentation and management in children and adults. Chest 115: 1357-1362. Link: https://bit.ly/2Pg51bw

15. Klein A, Ovnat-Tamir S, Marom T, Gluck O, Rabinovics N, et al. (2019) Fish Bone Foreign Body: The Role of Imaging. Int Arch Otorhinolaryngol 23: 110115. Link: https://bit.ly/32CBDzc

16. Zuluaga CP, Aluja Jaramillo $F$, Velásquez Castaño SA, Rivera Bernal AL, Granada JC, et al. (2016) Aortic Pseudoaneurysm Secondary to Mediastinitis due to Esophageal Perforation. Case Reports in Radiology 26977330. Link: https://bit.ly/3dGetOQ

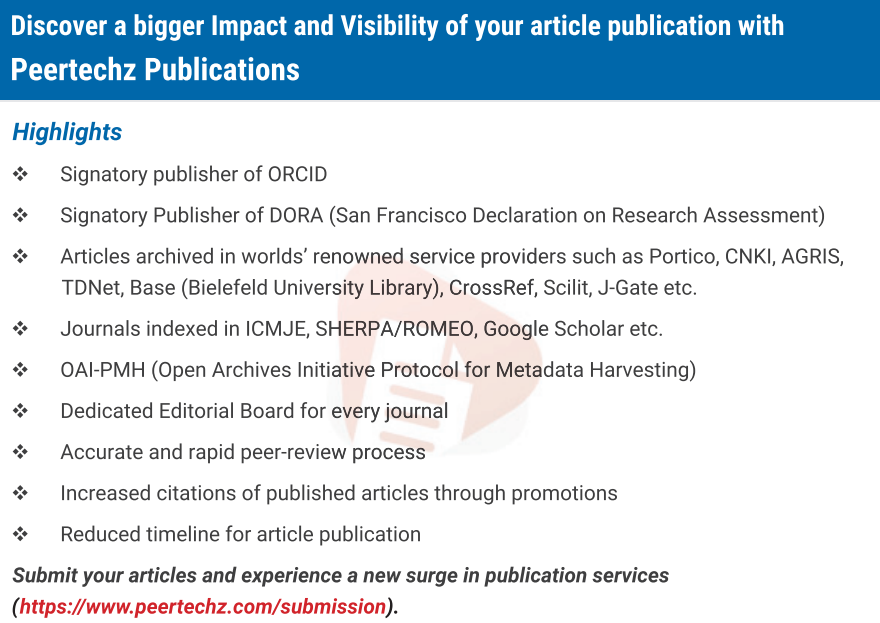

Peertechz journals wishes everlasting success in your every endeavours.

Copyright: @ 2021 Bagdadi RR, et al. This is an open-access article distributed under the terms of the Creative Commons Attribution License, which permits unrestricted use, distribution, and reproduction in any medium, provided the original author and source are credited. 